International Journal on Cybernetics \& Informatics (IJCI) Vol. 5, No. 4, August 2016

\title{
SHIFT INVARIENT AND EIGEN FEATURE BASED IMAGE FUSION
}

\author{
S.B.G.Tilak Babu ${ }^{1}$, V.Satyanarayana ${ }^{1}$ and Ch.Srinivasarao ${ }^{2}$ \\ ${ }^{1}$ Dept. of ECE,AEC, Surampalem,Kakinada, India \\ ${ }^{2}$ Dept. of ECE,JNTUK, UCEV,Vizianagaram, India.
}

\begin{abstract}
:
Image fusion is a technique of fusing multiple images for better information and more accurate image compared input images. Image fusion has applications in biomedical imaging, remote sensing, pattern recognition, multi-focus image integration, and modern military. The proposed methodology uses benefits of Stationary Wavelet Transform (SWT) and Principal Component Analysis (PCA) to fuse the two images. The obtained results are compared with exiting methodologies and shows robustness in terms of entropy, Peak Signal to Noise Ratio (PSNR) and standard deviation.
\end{abstract}

\section{KEY WORDS:}

Fusion, multi-focus image integration, SWT, PCA.

\section{INTRODUCTION}

Image fusion is for obtaining more accurate information by combination of two or more images. The fusion has many applications like biomedical imaging, air traffic control, robotics, remote sensing, pattern recognition, multi-focus image integration, and modern military environmental monitoring [1].

The image fusion can be achieved in spatial domain or frequency domain. Jiayi Ma et al., proposed a technique of fusing infrared image and visible image. The fusion of two images is depended on Gradient Transfer Fusion (GTF), GTF is a combination of gradient transfer with minimization of total variation. Jiayi Ma et al., are succeeded image fusion without any registration of source images [5]. Yanfei and Nong [6] proposed a multi sensor image fusion technique based on hierarchical multi resolution along with attention. Important areas are identified by using visual attention model and maximum entropy. Based on adoptive weighing rules, first level of fused image is obtained from visible image and infrared image. Finally Non Subsampled Counterlet Transform (NSCT) is used to obtain final fused image. Huafeng Li [7] also proposed for the fusion of multi sensor image combination based on NSCT. Jun Lang and Zhengchao image fusion technique [8] provides less spectral distortion and good spatial resolution based on discrete fractional random transform and adaptive pulse coupled neural network $(\mathrm{PCNN})$.

Principal Component Analysis (PCA) [2] increases the spatial resolution and the flaw is creation of distortion in spectrum in remote sensing image fusion. SWT advantage translation invariance over DWT and DWT advantage is time frequency localization. The paper set as follows, in section 2 proposed methodologies, its flow diagram and information about techniques used DOI: $10.5121 /$ ijci.2016.5418 
presented. In section 3, results and performance calculations and analysis are presented. Finally conclusions along with future directions are given in section 4 .

\section{Proposed Method}

The proposed technique uses the advantage of SWT and PCA. Initially, the input images of any size are registered into same size, and then given to SWT separately. The SWT converts an image into four different subbands A, H, V and D. In figure 1 A1 and A2, H1 and H2, V1 and V2, D1 and D2 are approximate coefficients, horizontal details, vertical details, diagonal details of image 1 and image 2 respectively. The approximate coefficients are fused based on PCA, similarly horizontal details, vertical details and diagonal details also fused individually. After fusion of respective coefficients, the fused coefficients are given to ISWT, which results final required fused image.

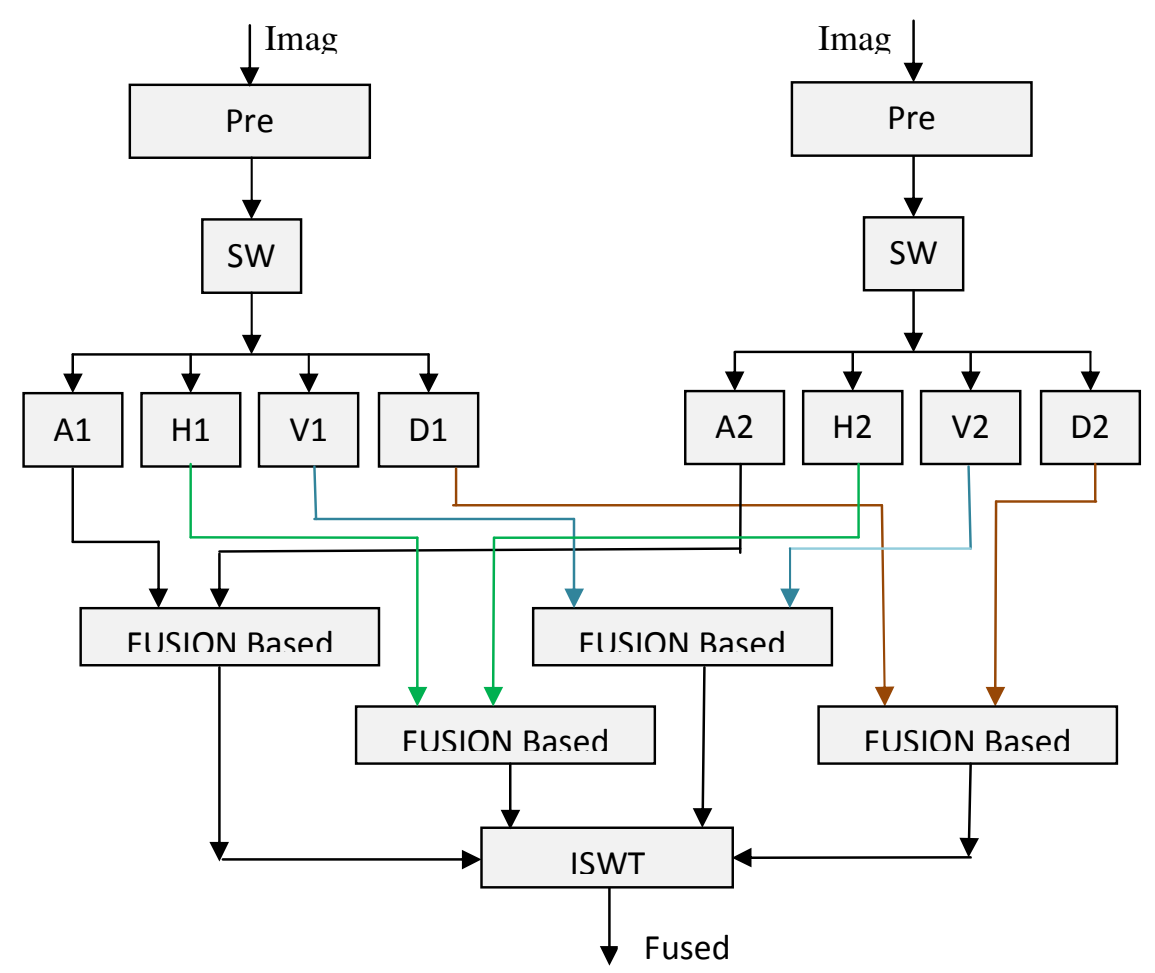

Fig1: Flow diagram of proposed method

\subsection{Stationary Wavelet Transform}

The drawback in discrete wavelet transform is translation invariance is overcomes in stationary wavelet transform. Due to presence of up-samplers and down-samplers in DWT, it lacks translation invariance and this effect is eliminated in SWT by removing the up-samplers and down-samplers, so SWT also called as Translation invariant wavelet transform. The outcome of SWT consist same number of samples as the input, so it is also called redundant wavelet transform. 


\subsection{Principal Component Analysis}

PCA is a statistical procedure based on orthogonal transformation; to convert set of structure of possible correlated variables into set of linearly uncorrelated variable called "principle components". Output after PCA consists of less number of Eigen features when compared to original input spectral feature. In this method initially need the mean values which is given in Equation (1) of spectral features later Calculate the covariance matrix in Equation (2), based on this covariance matrix find the feature vectors. To find out the Eigen features by using singular value decomposition (SVD) method in Equation (3).

$$
\begin{gathered}
E(X)=\frac{1}{k} \sum_{i=1}^{k} x_{i} \\
\operatorname{Cov}_{i}(X)=\left(\mathrm{X}_{\mathrm{i}}-\mu\right)\left(\mathrm{X}_{\mathrm{i}}-\mu\right)^{\mathrm{T}} \\
A=U \sum V^{T}
\end{gathered}
$$

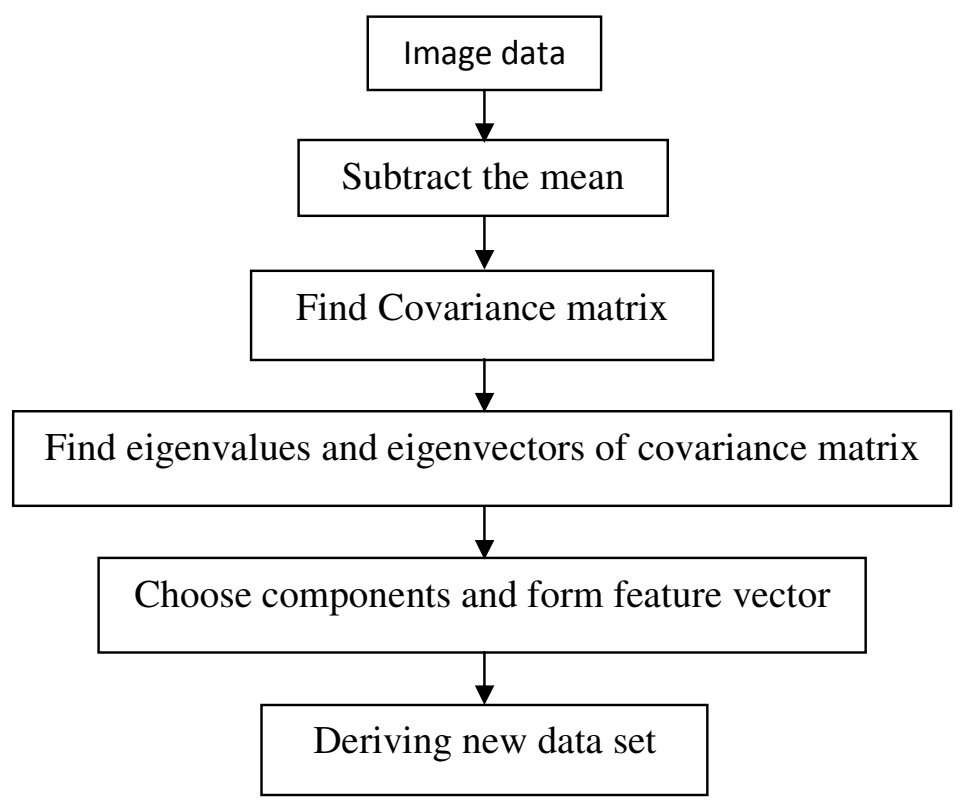

Fig.1 Flow diagram of PCA

\section{Results}

The results of proposed methodology are displayed in below tables and here the testing database downloaded from standard organizations [9], [10]. Table 1 displays, proposed method results from dataset 1 [10].Table 2 displays, proposed method results from dataset 2 [9]. All the process done on personal computer with RAM 2GB, Matlab version 2013. 
International Journal on Cybernetics \& Informatics (IJCI) Vol. 5, No. 4, August 2016

Table1: Comparison of Fused results for dataset1.

\begin{tabular}{|l|l|l|l|l|l|l|}
\hline \multirow{2}{*}{ ImagelMeasurement } & \multicolumn{3}{|l|}{ ENTROPY } & \multicolumn{3}{l|}{ STANDARD DEVIATION } \\
\cline { 2 - 7 } & PCA & SWT & PCA+SWT & PCA & SWT & PCA+SWT \\
\hline Pair1(DU) & 6.25 & 6.99 & 7.40 & 25.62 & 25.98 & 29.73 \\
\hline Pair2(NU) & 6.08 & 6.59 & 7.43 & 11.99 & 13.32 & 15.70 \\
\hline Pair3(FLY) & 4.66 & 4.65 & 5.68 & 41.87 & 42.19 & 41.89 \\
\hline
\end{tabular}

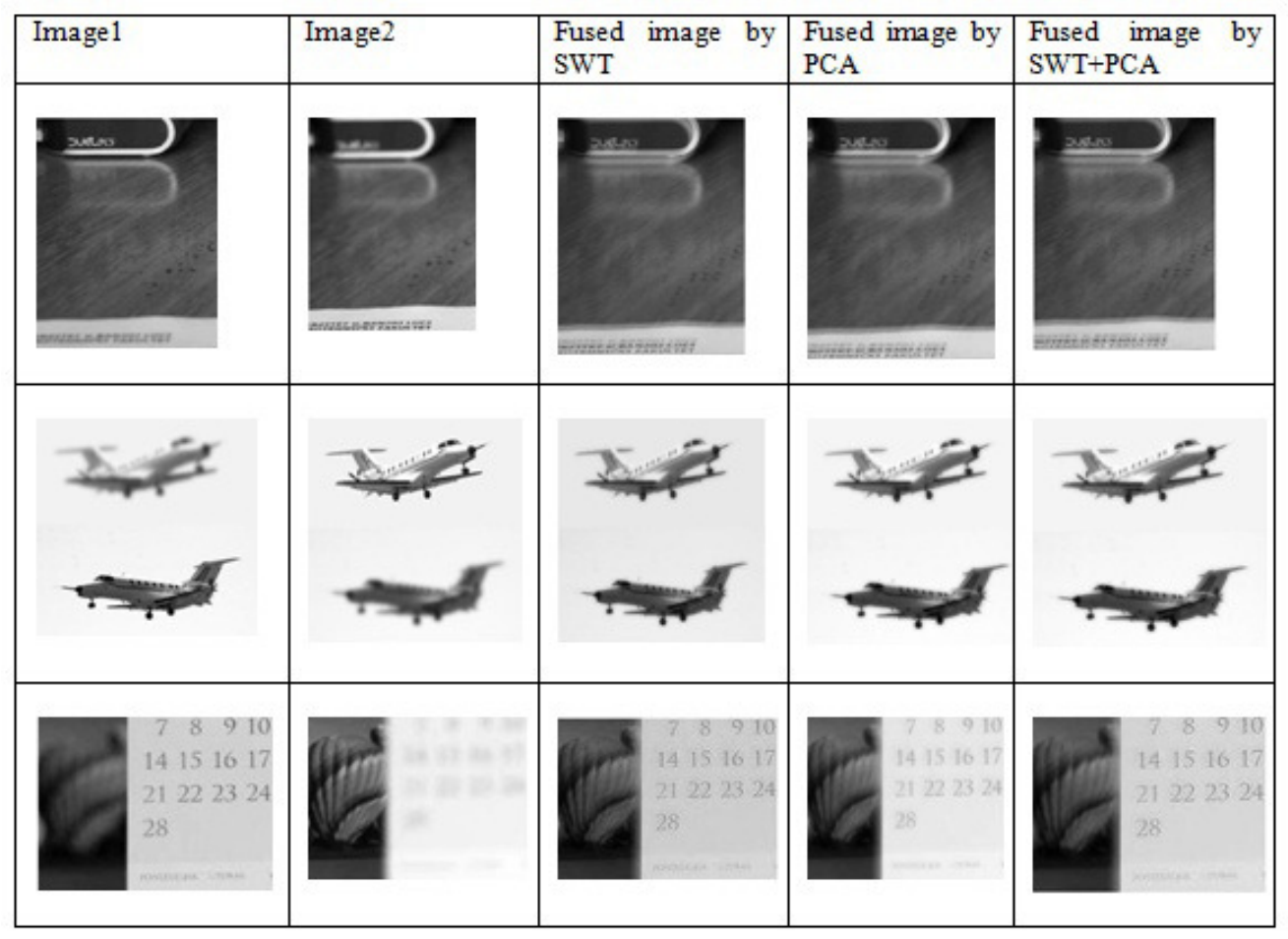

Table2: Comparison of Fused results for dataset2. 
International Journal on Cybernetics \& Informatics (IJCI) Vol. 5, No. 4, August 2016

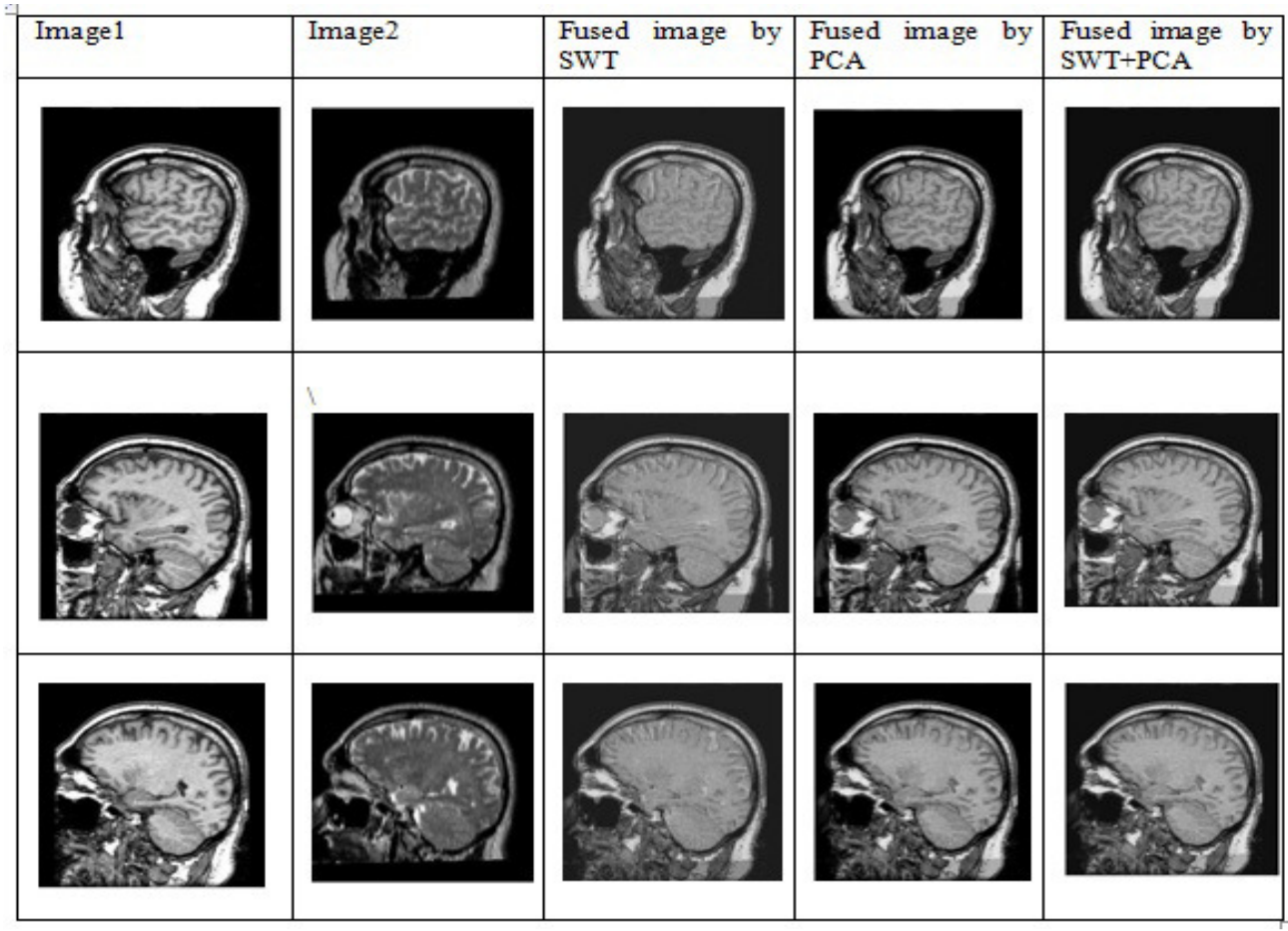

Table3: Performance calculations comparison in PSNR.

\section{Performance Calculations}

For testing of proposed methodology, PSNR, Standard Deviation and Entropy are used [4], [3]. The performance calculations are presented in below table3 and table4. PSNR1 represents for peak signal to noise ratio of fused image with image1 and PSNR2 for peak signal to noise ratio with image2.

\begin{tabular}{|l|l|l|l|l|l|l|}
\hline \multirow{2}{*}{ ImagelMeasurement } & \multicolumn{3}{|l|}{ PSNR 1 } & \multicolumn{2}{l|}{ PSNR 2 } \\
\cline { 2 - 7 } & PCA & SWT & PCA+SWT & PCA & SWT & PCA+SWT \\
\hline Pair1(DU) & 23.00 & 22.86 & 33.60 & 21.20 & 21.19 & 31.80 \\
\hline Pair2(NU) & 20.84 & 27.00 & 39.27 & 23.29 & 17.21 & 38.98 \\
\hline Pair3(FLY) & 26.65 & 24.19 & 38.45 & 26.70 & 24.23 & 36.99 \\
\hline
\end{tabular}

Table4: Performance calculations comparison in Entropy and Standard Deviation.

\section{Conclusions}

The proposed fusion methodology is done by the help of SWT and PCA, SWT has the advantage of shift invariance over Discrete Wavelet Transform. The comparative analysis of performance calculations showing robustness over remaining two techniques. The future idea is to fuse infrared images. 
International Journal on Cybernetics \& Informatics (IJCI) Vol. 5, No. 4, August 2016

\section{REFERENCES}

[1] P. K. Varshney, "Multisensor data fusion," in Electronics \& Communication Engineering Journal, vol. 9, no. 6, pp. 245-253, Dec 1997.

[2] Deepak Kumar Sahu, M.P.Parsai, "Different Image Fusion Techniques -A Critical Review" International Journal of Modern Engineering Research (IJMER), Vol. 2, Issue 5, pp 4298-4301, SepOct 2012.

[3] Shrivsubramani, Krishnamoorthy, K P Soman,“ Implementation and Comparative Study of Image Fusion Algorithms”. International Journal of Computer Applications (0975 - 8887) Volume 9- No.2, November 2010.

[4] V.P.S. Naidu, J.R. Raol, "Pixel-level Image Fusion using Wavelets and Principal Component Analysis”. Defence Science Journal, Vol. 58, No. 3, May 2008, pp. 338 -352, 2008.

[5] Jiya ma, Chen Chen, Chang Le, Jun Huang, "Infrared and visible image fusion via gradient transfer and total variation minimization" Information Fusion, Elsevier, pp. 100-109, 2016.

[6] Yanfei Chen, Nong Sang , "Attention-based hierarchical fusion of visible and infrared images", Volume 126, Issue 23, PP 4243-4248, August 2015.

[7] Xiaosong Li , Huafeng Li , Zhengtao Yu , Yingchun Kong, "Multifocus image fusion scheme based on the multiscale curvature in nonsubsampled contourlet transform domain", Volume 54, Issue 7, Imaging Components, Systems, and Processing, Jul 30, 2015.

[8] Jun Lang, Zhengchao Hao, "Image fusion method based on adaptive pulse coupled neural network in the discrete fractional random transform domain", International Journal for Light and Electron Optics, Elesiver, Volume 126, Issue 23, Pages 3644-365, December 2015.

[9] Keith A. Johnson, J. Alex Becker, “ http://www.med.harvard.edu/aanlib/home.html”, The whole brain atlas data set, image database1.

[10] Slavica Savic, "http://dsp.etfbl.net/mif/", Image database2

\section{AUTHOR}

S.B.G.Tilak Babu received his Bachelor's degree in Electronics and Communication Engineering from SVIET, Nandamuru and Master's from JNTUK University college of Engineering Vizianagaram in 2013 and 2015 respectively. He joined as an Assistant Professor in Aditya Engineering College, Surampalem in 2015. His research interest includes digital signal processing, speech processing, audio processing, image, video processing and soft computing techniques.

V.Satyanarayana received his Bachelor's and Master's degree in Electronics and Communication Engineering from Aditya Engineering College, Surampalem. His research interests are Digital Image Processing and VLSI. He is a member of IETE. Presently, he is working as an Associate Professor in the Department of ECE, Aditya Engineering College, Surampalem, A.P., India.



Ch.Srinivasarao received his Bachelor's degree in Electronics and Communication Engineering from DMSSVHCE, machilipatnam and his Master's and PhD from JNTU Kakinada. He's working as a professor in JNTUK University college of Engineering Vizianagaram, A.P., India. He published 40 research articles in reputed national and international journals. His research interest includes digital signal processing, speech processing, audio processing, image processing, video processing and soft computing techniques.
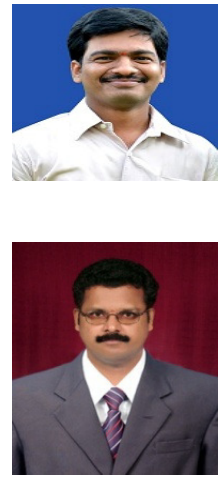